\title{
Study on mechanical properties and microstructure analysis of welded joints of 7A05 aluminum alloy by laser-MIG hybrid welding
}

\author{
Hongwei $\mathrm{Liu}^{1,2, \mathrm{a}}$, Jinglong $\mathrm{Li}^{1, \mathrm{~b}}$, Zhihua $\mathrm{Ma}^{2, \mathrm{c}}$, Bing $\mathrm{Ma}^{2, \mathrm{~d}}$ and Shengqiang \\ Feng $^{2, \mathrm{e}}$ \\ ${ }^{1}$ Shanxi Key Laboratory of Friction Welding Technologies,Northwestern polytechnical \\ University,Xi'an 710072,China \\ ${ }^{2}$ Ningbo Branch of China Academy of Ordnance Science,Ningbo 315103,China \\ a13586517687@139.com, b lijinglg@nwpu.edu.cn, c mazhihua0301@163.com, \\ d mabing_008@hotmail.com, ${ }^{\text {e }}$ fsq7980@tju.edu.cn
}

Keywords: 7A05 aluminum alloy, laser-MIG hybrid welding, mechanical properties, microstructure analysis

\begin{abstract}
A05 aluminum alloy joints were fabricated by Laser-MIG hybrid welding, of which the tensile strength was tested at room temperature. The tensile fracture surfaces and microstructure were analyzed by SEM and TEM. The fracture surface analysis showed fracture morphologies of small dimple of $2 \sim 5 \mu \mathrm{m}$ on the heat affected zone (HAZ) and big dimple of $30 \sim 50 \mu \mathrm{m}$ on the weld bead. The fracture is inter-granular fracture with a small amount of cleavage fracture. TEM analysis showed that the HAZ region didn't exists $\eta$ '-phase, but $\mathrm{Al}_{3} \mathrm{Zr}$, i.e., during welding thermal cycle, the joints appeared obvious $\eta$ '-phase back dissolved so that lost $\eta$ '-phase dispersion strengthening effect. $\mathrm{O}, \mathrm{Na}$ and $\mathrm{Fe}$ impurities were detected in the joint, of which the crack originated during tensile testing. Both the impurities and $\eta$ '-phase back dissolved are responsible for the degrading of the welded joints mechanical properties.
\end{abstract}

\section{Introduction}

Aluminum alloy is widely used in the fields of aerospace, automobile manufacturing, shipbuilding and chemical containers for its high specific strength, corrosion resistance, and excellent machining performance. 7A05 aluminum alloy is a kind of Al-Zn-Mg weldable aluminum alloy. It has excellent mechanical properties and weldability, which is the main load-bearing member in the military field and the bridge structure ${ }^{[1,2]}$. When using gas tungsten arc welding (TIG) and metal inert gas shielded welding (MIG), the joint heat affected zone (HAZ) normally softens owing to large quantity of heat input and the weld bead tends to form porosity structure, all of which would ruin the joint performance ${ }^{[3,4]}$. Thus, more concentrative heat source such as laser beam is welcome. However, aluminum surface reflects most of the laser input.

Laser-MIG hybrid welding integrates heat sources of both laser and MIG that has the advantages of weld penetration, high efficiency, small deformation, lower assembly requirements and better bridging ability of the pool. Laser-MIG welding can overcome the shortcomings existing in TIG or MIG welding of aluminum alloy. Recently, research on Laser-MIG hybrid welding technology is highlighted for the potential industrial production along with the rapid development of welding methods ${ }^{[5-7]}$. However, laser-MIG hybrid welding on 7A05 aluminum alloy has rarely reported. In this study, 7A05 aluminum alloy joint was made by Laser-MIG hybrid welding. The joint strength was evaluated and the joint structure was analyzed.

\section{Experiment}

Commercially available 7A05 aluminum alloy sheet was used as substrate that was prepared for welding in the size of $300 \mathrm{~mm} \times 150 \mathrm{~mm} \times 10 \mathrm{~mm}$. Prior to welding, the plate was supersonic washed by acetone to clean the grease film on the surface of the plate, which was then rinsed in water and 
dried in a oven. At last, the oxide film on the surface was removed by a polishing machine. ER5356 aluminum wire with a diameter of $1.2 \mathrm{~mm}$ was selected as filler metal. A DC $050 \mathrm{CO}_{2}$ slab laser (ROFIN Company) was used as the laser source. The diameter of focus spot was about $0.45 \mathrm{~mm}$ by using 300mm focus lens. Invision 456MP MIG welder (Miller Comany) was used as the arc heat source. The welding shielding gas consists of argon and helium (Ar: $\mathrm{He}=10: 15$, volumetric ratio). Two heat sources were integrated by using paraxial composite mode, in which the laser was set in the front and the arc in the rear.

Tensile test at room temperature was carried out both on welded joints and base metal and the fracture morphologies were observed by scanning electron microscope (SEM).

Transmission electron microscope (TEM) was further used to clarify structures and phases, in which Fig.1 presents sampling locations, i.e., the center of the weld bead (1\#), HAZ (2 \#) and 7A05 base metal $(0 \#)$. The samples were prepared by mechanical pre-thinning and chemical double spray thinning, in which the electrolyte reagent is a mixture composed of nitric acid and methanol (volumetric ratio 1:3) and the temperature of the electrolytic thinning was set below $-25^{\circ} \mathrm{C}$.

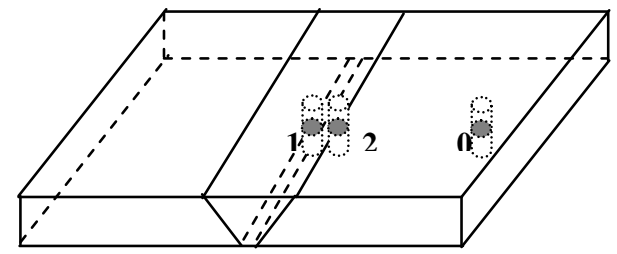

Fig. 1 TEM sampling locations

\section{Experimental results and analysis}

\section{Tensile mechanical properties at room temperature}

Table 1 shows the joint tensile testing results of the joint and substrate for comparison. The results show lower strength of welded joints, among which most fractured in the welded joint. The joint tensile strength decreased from $425 \mathrm{MPa}$ to $285 \mathrm{MPa}$ resulting in a strength coefficient as $67 \%$. Whereas, the yield strength dropped from 390MPa to 210MPa. Although it is higher than the gas tungsten arc welding and metal inert gas shielded welding, low strength is quite obvious.

Tab. 1 Tensile properties of the substrate and the welded joint of laser-MIG 7A05 plate

\begin{tabular}{lcccc}
\hline \multirow{2}{*}{ test sample } & \multicolumn{2}{c}{ tensile properties } & \multicolumn{2}{c}{ Welded joint } \\
& $\sigma_{\mathrm{b}} / \mathrm{MPa}$ & $\sigma_{0.2} / \mathrm{MPa}$ & $\delta_{5} / \%$ & strength coefficient \\
\hline substrate & 425 & 390 & $13.5 \%$ & $67 \%$ \\
welded joint & 285 & 210 & $3.1 \%$ & \\
\hline
\end{tabular}

\section{The hardness distribution of the welded joints}

Fig. 2 shows the micro Vickers hardness distribution of welded joints. The micro Vickers hardness measurement points sequentially through the base material 7A05, the HAZ and the center of the weld region, and the weld centerline as the origin of the coordinate.

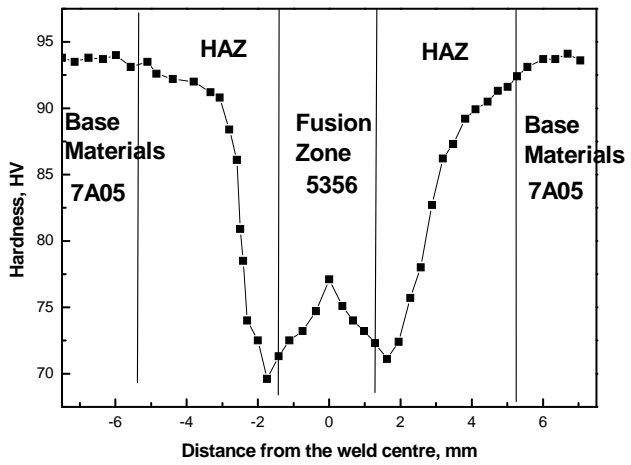

Fig. 2 Vickers hardness curve across the laser-MIG weld of 7A05 plate 
The results show that the hardness of the welded joints is approximately symmetrical distributed about the weld centerline, where the mean hardness of the welded joints is lower than base metal. With the increase of the distance from the center of the weld, the hardness showed a rapid increase till the hardness of the substrate. Generally, the yield strength is proportional to the hardness of the aluminum alloy. This result is consistent to the loss of yield strength as compared in Table 1.

One may notice that the severe softening happened at HAZ near the fusion line that presents lowest value of hardness, in which structural change may occur during the welding thermal cycle.

\section{Fracture surface analysis}

In macroscopic view, plastic deformation on the fracture surface is quite few. However, dimple structures with cleavage crack were found as shown in Fig.3. 60\% area of the fracture surface exhibits obvious dimple pattern as shown in Fig $3 \mathrm{a}$. The size of the dimples varies a lot. The bigger ones are $30 \sim 50 \mu \mathrm{m}$, while the smaller ones are $2 \sim 5 \mu \mathrm{m}$ as shown in Fig $3 \mathrm{~b}$. In the subsequent TEM analysis, the size of bigger and smaller dimples are corresponded to the grain sizes in weld center and HAZ respectively, which confirms that cracks originated from both HAZ and weld center and resulted in various dimple size. All dimples are of parabolic shape, which means that the crack mode of the joint is mainly inter-granular crack.

Except for the inter-granular crack, there was a little cleavage crack (Fig 3c). Some O, $\mathrm{Na}$ and $\mathrm{Fe}$ were found in the cleavage fracture surface by EDS as shown in Fig.3d. The brittle impurity composed by these elements that probably added into the joint during the welding operation which tended to generate more micro cracks and consequently degraded the tensile strength.

Although HAZ presented lowest hardness, it does not mean to conclude that the HAZ is the area where crack originates because the crack initiation and crack propagation matter a lot in the tensile failure process. It is clear that the impurity in the bonding area of HAZ and 5356 alloy (i.e., weld bead) induced micro cracks. These cracks extended into both HAZ and welding bead and later caused different dimple patterns on the fracture surfaces after the tensile tests.

Although hardness of the HAZ is lowest according to micro-hardness analysis, it is difficult to come to a conclsion that the HAZ is the area where crack originates because the crack initiation and crack extension matter a lot in the tensile failure process. The impurity in the bonding area of HAZ and 5356 alloy induced micro cracks. These cracks extended into HAZ and welding seam and caused different $\mathrm{s}$

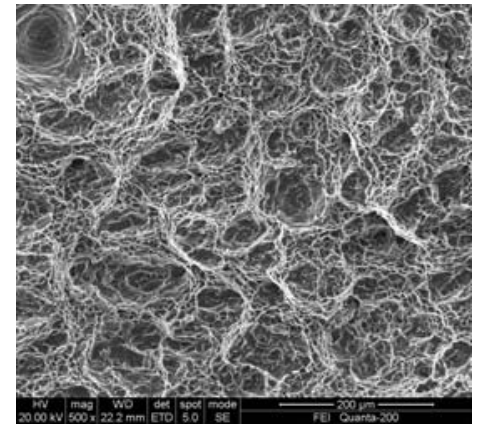

(a) Obvious dimple pattern

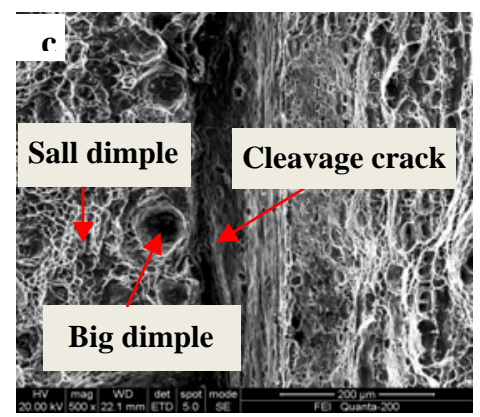

(c) Cleavage fracture morphology

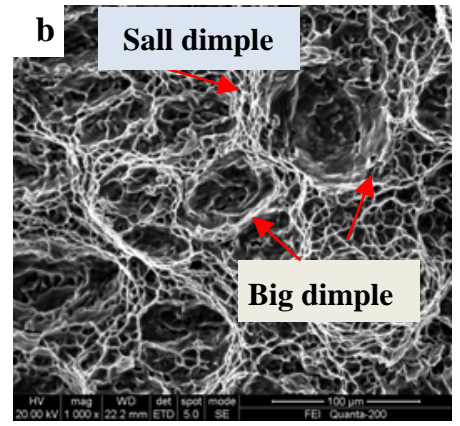

(b) Uneven Size dimple

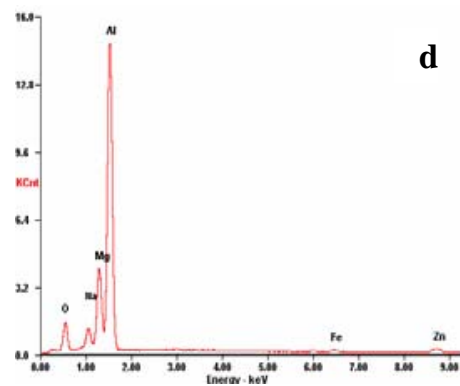

(d) EDS diffraction patterns

Fig. 3 Fractographs of tensile-tested laser-MIG weld of 7A05 joint 


\section{Microstructure transformation of the welding joint}

\section{Microstructure of 7A05 substrate}

Fig 4 shows TEM patterns of 7A05 base metal. The substrate grain retained elongated morphology as shown in Fig.4a. The grain elongation is not obvious because of the small rolling deformation of the plate. Therefore, the microstructure was not refined homogeneously by the welding thermal cycle. The mean grain size is $2 \sim 5 \mu \mathrm{m}$. There are a lot of sub-grain boundaries composed of dislocations. Whereas only a few dislocations existed inside of the grain, which means that full reversion has occurred in the aging process of the plate before welding. Dislocations formed sub-boundaries through slipping as shown in Fig. 4b. There are many dispersive phases with diameter of $2 \mathrm{~nm}$ precipitated in the grain. Fig.5 shows diffraction pattern of these dispersive phases that are $\eta^{\prime}$-phase. Bean-like $\mathrm{Al}_{3} \mathrm{Zr}$ phase, though visible in the substrate as shown in Fig.4, is not measured in the diffraction pattern because of its low content.



(a) Rolling residue elongated grain morphology

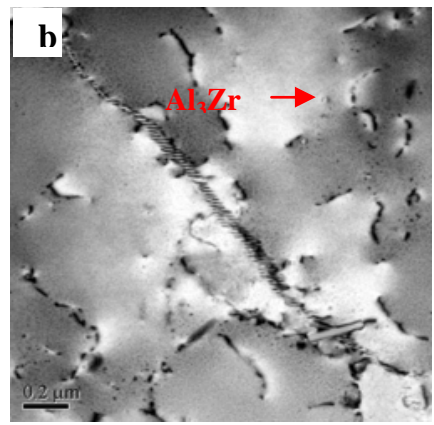

(b) Dislocation glide formation of sub-boundaries

Fig. 4 7A05aluminum alloy microstructure

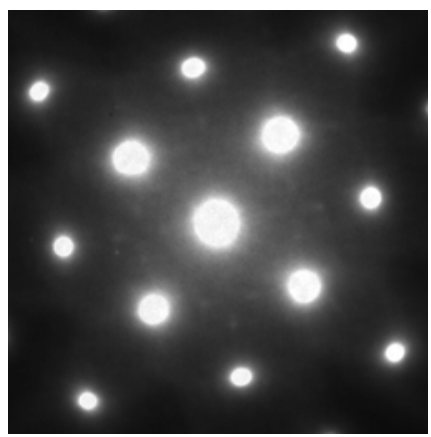

Fig. 5 Electronic diffraction of 7A05 parent material

\section{Microstructure of HAZ}

Fig.6 shows HAZ microstructure of the joint. Grain size and dislocation density of HAZ do not vary much compared with 7A05 substrate by Fig.6a and Fig.4a. The sub-grain boundary is mainly in reversion state as shown by Fig. $6 \mathrm{~b}$; whereas recrystallization partly takes place in the HAZ as indicated in Fig.6c.

TEM analysis shows there is no $\eta^{\prime}$-phase in $\mathrm{HAZ}$ but $\mathrm{Al}_{3} \mathrm{Zr}$ as shown in Fig. 6d, which means $\eta^{\prime}$-phase has dissolved into matrix of HAZ during welding thermal cycle. Without the dispersion strengthening of $\eta^{\prime}$-phase, the mechanical properties (tensile strength and yield strength) of the joint degraded consequently.

The high strength of $\mathrm{Al}-\mathrm{Zn}-\mathrm{Mg}-\mathrm{Cu}$ alloy is attributed to the precipitation of $\eta^{\prime}$-phase during its quenching and tempering process. The precipitation sequence is as follows: SSS (saturated solid solution) $\rightarrow$ GP (I, II) domain $\rightarrow \eta^{\prime}$-phase. In the welding process, the temperature field is axisymmetric about the weld center line. The $\eta^{\prime}$-phase adjacent to the weld center tends to dissolve significantly as which exposes higher temperature. Correspondingly, hardness and strength decrease more severely, which is in coincidence with the micro hardness analysis.

The solid solubility of $\mathrm{Zr}$ in $\mathrm{Al}$ is $0.28 \%$ in equilibrium state, whereas it is $0.35 \%$ during quenching process of semi-continuous casting. Certain amount of $\mathrm{Zr}$ can help to suppress re-crystallization and coarsening because of dispersive fine precipitation of Al3Zr. Generally, phase structure of $\mathrm{Al} 3 \mathrm{Zr}$ is 
relatively stable as its melting point is as high as $1580^{\circ} \mathrm{C}$. It is difficult for them to dissolve into the matrix during heat treatment. So the welding thermal cycle influences Al3Zr little, i.e., there is no
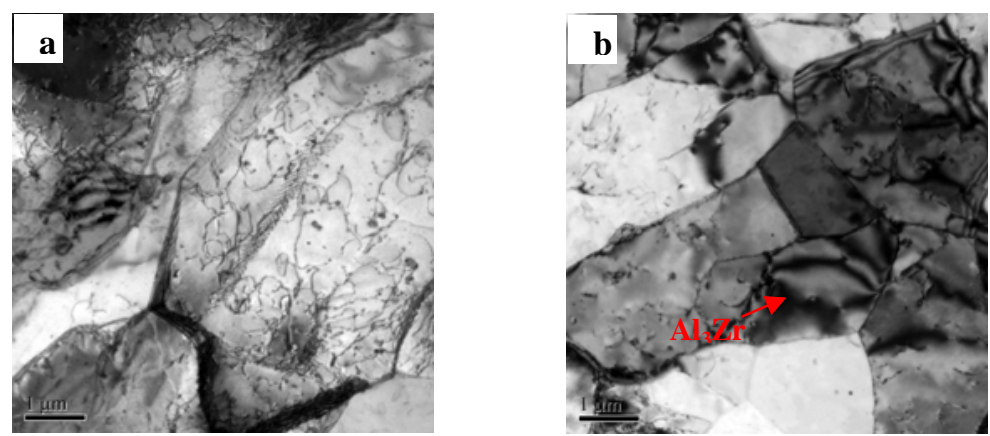

decomposition or coarsening happened in the welding process. Thus, its suppression on re-crystallization made the substrate preserve the original microstructure.
(a) Heat-affected zone grain
(b) Dislocation subgrain boundaries

(c) Recrystallized structure

(c) High magnification TEM structure

Fig. 6 Bright field microstructure image of HAZ of 7A05 alloy

\section{Microstructure of the weld bead}

Fig. 7 shows the microstructure of the weld bead (fusion zone). The composition of the weld bead is a mixture of 5356 filler metal and 7A05 substrate, which are melted and mixed by the laser-arc heat source and later solidified to form casting structure. The grain size is as big as $20 \mu \mathrm{m}$, which corresponds to the big dimples observed on fracture surface as shown in Fig.3.

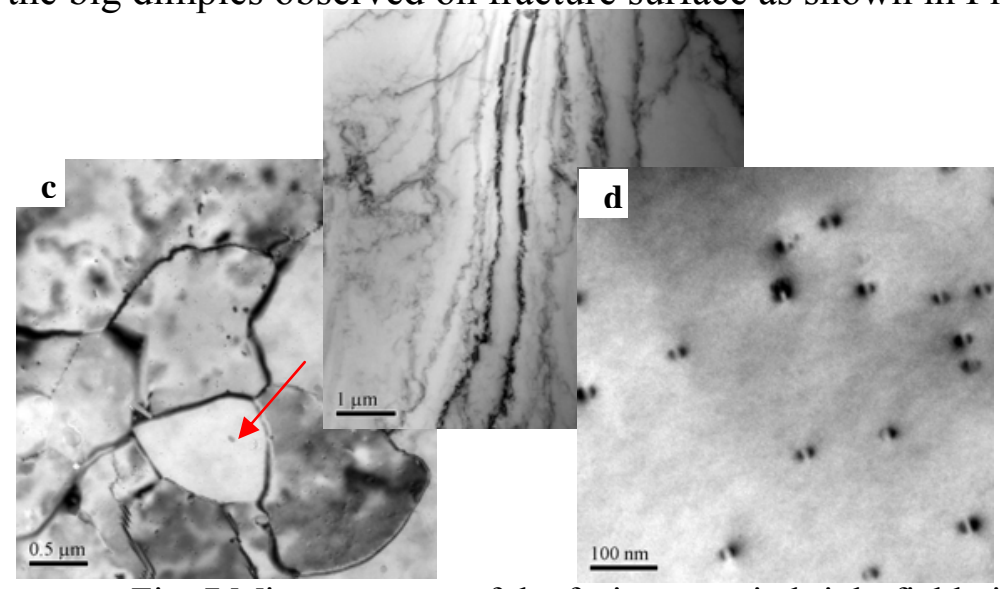

Fig. 7 Microstructure of the fusion zone in bright field view

\section{Conclusions}

1) Tensile strength of Laser-MIG welding joint of 7A05 alloy is quite poor, in which the strength coefficient is only $67 \%$. Certain amount of $\mathrm{O}, \mathrm{Na}$ and $\mathrm{Fe}$ impurities were detected in the welding joint, which is responsible for the degrading of the mechanical properties.

2) During tensile testing, Crack originated in impurities distributed in the bonding area of HAZ and weld bead and further expanded into HAZ and weld center.

3) The dissolution of $\eta^{\prime}$-phase into the matrix softens HAZ, which is also responsible for the degrading of the mechanical properties.

\section{References}

[1] T.Wang, Z.M.Yin. Chinese Journal of Rare Metals.Vol.30(2006), p16.

[2] W.S.ZHOU, J.S.YAO. Welding of aluminum and aluminum alloy(Machinery Industry Press,Beijing2006).

[3] L.H.XU, Z.L.TIAN, Y.PENG,etc. Transactions of the China welding institution, Vol.28(2007),p39-42. 
[4] D.G.CHEN,H.W.LIU,B.TAN,etc.ordnance material science and ngineering.Vol.32(2009), p55-57.

[5] G. Casalino. Statistical Analysis of MIG-laser CO2 Hybrid Welding of Al-Mg alloy. Journal of Materials Processing Technology, Vol.191(2007),p106-110.

[6] T Ueyama, H Tong, I Yazawa. Welding International. Vol.18(2004), p345-350.

[7] S. B. Hee, S. B. Han, C. k. You, et al. Materials and Design. Vol.32 (2011), p2328-2333. 